\title{
Fiber-Optic Liquid-Interface Sensor for Liquid Hydrogen
}

\author{
Sergei Khotiaintsev*, Volodymyr Svyryd \\ and Heidy B. Mejia del Puerto \\ Faculty of Engineering, National Autonomous University of Mexico, \\ Ciudad Universitaria, c. p. 04510, Mexico, D. F., Mexico
}

(Received January 16, 2008; accepted September 17, 2008)

Key words: fiber-optic sensor, optical refractometry, liquid-interface sensor, liquid-level measurement, liquid hydrogen, cryogenics

We describe a refractometric fiber-optic sensor, which was developed and successfully used for the detection of the liquid interface in liquid-hydrogen storage tanks. The sensor employs a small hemispherical detection element of fused silica, which is integrated with two multimode optical fibers. The sensor parameters are optimized for the reliable discrimination between two media of almost equal refractive indices: liquid hydrogen and gaseous hydrogen. We show theoretically and experimentally that the dimensions of the sensor can be reduced without sacrificing its sensitivity if optical fibers with a small angular aperture are used and the position of the fibers results in a significant loss of non-liquid-dependent light. The advantages of this sensor in comparison with electrical sensors are the smaller heat ingress in the liquid-hydrogen tank, its intrinsic safety, and its potentially low cost. This sensor can be used as a point device, in pairs, or in multipoint arrays, such as those used in discrete liquid-level sensors. Its prospective applications include liquid-hydrogen-level sensing in hydrogen-powered cars, aircraft, space vehicles, and liquid-hydrogen production and storage facilities.

\section{Introduction}

Liquid hydrogen (LH2) is widely used as a propellant in space vehicles. ${ }^{(1)}$ This application is expected to continue to grow in the future due to such programs as the International Space Station and space tourism. Also, LH2 has prospective applications in terrestrial automotive systems and aircraft. A large amount of research has been carried out on different concepts of hydrogen-powered vehicles in recent years. ${ }^{(2,3)}$ Hydrogen-powered concept cars and buses have already been demonstrated. Therefore, the production of liquid hydrogen and its use in various vehicles is expected to grow during the next decade. Level indicators and level sensors are important elements of any

*Corresponding author: e-mail: sergeikh@hotmail.com 
liquid-hydrogen storage system. Although various types of sensor have been proposed for the level detection and level measurement of cryogenic fluids and liquid hydrogen in particular, ${ }^{(4-7)}$ there is considerable interest in new systems.

In our previous work, ${ }^{(8)}$ we described a refractometric sensor consisting of a transparent detection element with a hemispherical working surface integrated with two multimode optical fibers. This sensor exhibits very different refractometric characteristics under various combinations of its geometrical parameters and material constants. ${ }^{(9)}$ Therefore, the basic optical design should be adapted to each particular application. One particular version of this sensor was used for the discrimination between a gas and a liquid near room temperature, ${ }^{(10)}$ and another version was used in a system that measured the level of liquid nitrogen. ${ }^{(11)}$ However, the refractive index $n$ of liquid hydrogen $(n=1.10974$ at wavelength $\lambda=590 \mathrm{~nm}$ and temperature $T=20$ $\mathrm{K})$ is significantly smaller than that of liquid nitrogen $(n=1.205$ at $\lambda=546 \mathrm{~nm}$ and $T$ $=83 \mathrm{~K}) \cdot{ }^{(12)}$ This makes the detection of the liquid interface in liquid-hydrogen storage tanks more difficult than in the case of a liquid-nitrogen interface. Also, the lower temperature of liquid hydrogen in comparison with liquid nitrogen and the flammability of liquid hydrogen mean that a more complex storage system is required than those used for liquid-nitrogen storage. This, in turn, results in a more complex arrangement of the optical fibers used to access the interior of the storage tanks, a larger number of gas-tight couplings, which may induce additional optical loss, and other complications.

In this paper, we describe a fiber-optic refractometric sensor, which was developed for the detection of the liquid interface in liquid-hydrogen storage tanks.

\section{Materials and Methods}

\subsection{Choice of optical materials for the sensor}

The sensor has to withstand cooling and heating in a wide temperature range-from the ambient temperature down to about $20 \mathrm{~K}$. The optical structure of the sensor will withstand multiple temperature cycles if the thermo-induced stress in it is minimized. To achieve this, the sensor and the connecting optical fibers should be made of the same material. Silica glass is the only type of optical-fiber material that works well in a cryogenic environment. Fibers made from other materials, such as plastics, are not suitable for cryogenic temperatures. Therefore, the detection element of the sensor should also be made of silica glass, which has high mechanical strength at normal and cryogenic temperatures, and has high resistance to water and acids. Its low thermal conductivity is a significant advantage in the present application.

There are a variety of glasses of high silica content that are used for the fabrication of optical fibers. In the present work we consider the use of fused synthetic silica for both the detection element and the core of the optical fibers.

\subsection{Considerations for the optical design of the sensor}

Liquid hydrogen has the second lowest index of refraction among fluids, after liquid helium. For the reliable discrimination between the liquid hydrogen and the gas located above the liquid in the tank (gaseous hydrogen or helium), the optical refractometric 
sensor should be able to detect a small change in the external refractive index from $n=1.0$ (the gas) to $n \approx 1.1$ (liquid hydrogen) and vice versa. In intensity-type refractometric sensors, the maximum sensitivity to the external refractive index is achieved when the angle of incidence of the optical rays on the working surface of the detection element $\theta_{\mathrm{i}}$ is larger than the critical angle for the glass-air interface $\theta_{\mathrm{c} \text { air }}$ but does not exceed the critical angle for the glass-liquid interface $\theta_{\text {c liquid }}$.

Simple calculations using Snell's law ${ }^{(13)}$ show that assuming a sensor of fused silica ( $n$ $=1.45$ at $940 \mathrm{~nm}$ and $20 \mathrm{~K})$, ${ }^{(14)}$ a flat working surface, and gaseous hydrogen or helium as the external medium $(n \approx 1)$, the critical angle $\theta_{\text {c gas }}$ is $43.6^{\circ}$. When the external medium changes from the gas to the liquid hydrogen, the critical angle increases to $\theta_{\mathrm{cLH} 2}=49.8^{\circ}$ (we assume the operational wavelength of $940 \mathrm{~nm}$ and $n_{\mathrm{LH} 2}=1.106$ at $940 \mathrm{~nm}{ }^{(7,12)}$ ). The light launched at the working surface within a sector with angle $\Delta \theta=\left|\theta_{\mathrm{c} \mathrm{gas}}-\theta_{\mathrm{c} \mathrm{LH} 2}\right|=6.2^{\circ}$ undergoes total internal reflection when the sensor is in the gas and partial total reflection when the sensor is in the liquid hydrogen. Therefore, the intensity of the reflected light is a function of the external medium (its refractive index).

For a nonpolarized central ray of the sector $\Delta \theta$, the reflection coefficient $R$ changes from $R_{\text {gas }}=1$ to $R_{\mathrm{LH} 2}=0.124$. This corresponds to a decrease in the reflected light intensity, which is converted to an electrical signal at the sensor output, by a factor of $\mathfrak{R}$ $=R_{\text {gas }} / R_{\mathrm{LH} 2}=I_{\mathrm{r} \text { gas }} / I_{\mathrm{rLH} 2}=8.1$ giving $\mathfrak{R}=9.1 \mathrm{~dB}$. For an optical beam of finite diameter and divergence, the signal $\mathfrak{R}$ is an integral quantity, which may be different from its value calculated for the central ray.

The obtained values of $\Delta \theta$ and $\mathfrak{R}$ are significantly smaller than the corresponding quantities for liquids such as liquid nitrogen $\left(\Delta \theta=12.2^{\circ}\right.$ and $\left.\mathfrak{R}=12.6 \mathrm{~dB}\right)$ and even more so for water $\left(\Delta \theta=23^{\circ}\right.$ and $\left.\mathfrak{R}=18.9 \mathrm{~dB}\right)$. This makes the development of refractometric sensors for liquid hydrogen difficult. Moreover, the signal may decrease due to a film of liquid remaining on the sensor surface ${ }^{(8)}$ or degradation of some elements. That is why it is desirable to increase the signal strength as much as possible.

An increase in the sensitivity of the sensor to the variation of the refractive index of the external media can be achieved under serial reflections of light at the glass/externalmedia interface in the sensor with the spherical working surface $S$ shown in Fig. 1. In addition, the spherical surface has a focusing property, which allows the use of divergent optical beams in the sensor. The number of internal reflections in the sensor $m$ is related to the angle of incidence of the central ray of the beam at the surface $S$. In the case of two serial reflections, $\theta_{\mathrm{i} \text { central }}=45^{\circ}$, and in the case of three serial reflections, $\theta_{\mathrm{i} \text { central }}=60^{\circ}$, and so on. It follows from the previous analysis that only two serial internal reflections are possible in the sensor in the present case. A larger number of internal reflections at the working surface $(m \geq 3)$ would result in angles of incidence with $\theta_{\mathrm{i}}>>\theta_{\mathrm{cLH} 2}$ and a complete loss of the sensitivity to the external refractive index in the range of interest. A larger number of internal reflections and greater sensitivity could have been obtained using a sensor material with a larger refractive index, but we restricted the material to fused silica. The choice of parameter $m$ determines the dimensionless distance between the optical fiber and the sensor axis $\Lambda=L / R$. It follows from simple geometrical considerations that $\Lambda_{m=2}=0.707$.

When considering other parameters of the sensor, it is important to decrease 


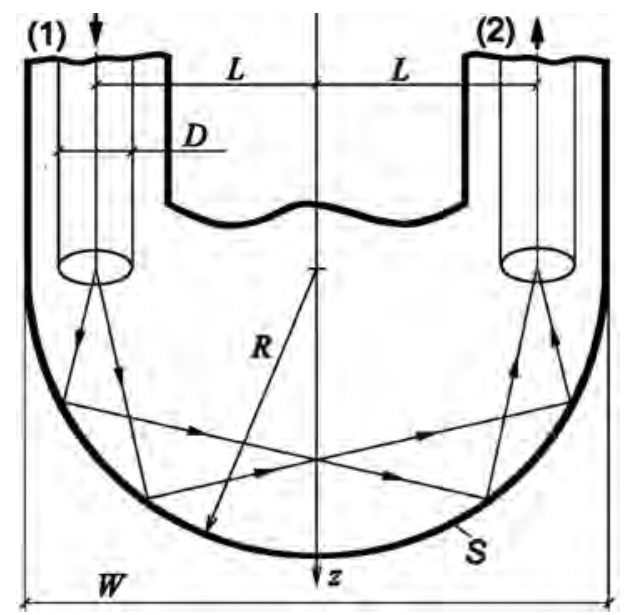

Fig. 1. Generic optical-fiber refractometric sensor with spherical working surface S. (1), (2) leadin and lead-out multimode optical fibers, respectively.

its transverse dimension $W=2 R$. In this way, the retention of liquid on its surface decreases. Also, miniature sensors can be used in tight places such as pipes and ducts. In view of these factors, it would be of practical interest to reduce the transverse dimension of the sensor $W$ to a fraction of a millimeter. Considering a typical diameter of the core of a multimode fiber, $D=50 \mu \mathrm{m}$, the transverse dimension $W \leq 1 \mathrm{~mm}$ corresponds to a dimensionless diameter of $\Phi \geq D / R=0.1$. That is, in practical cases the diameter of the core of the optical fiber $D$ is large in comparison with the radius of the detection element $R$. On the other hand, the angular aperture of realistic multimode optical fibers is significantly larger than the angular width of the sector $\Delta \theta$ obtained in this section. Therefore, the parameters obtained in this section are only estimated values. More precise results can be obtained using three-dimensional mathematical models of the sensor and numerical ray-tracing algorithms. In practice, the elaboration of the sensor is carried out by means of an iterative procedure comprising the design, analysis, and optimization.

\subsection{Procedure of numerical analysis}

The analysis of light propagation in the sensor was carried out by numerical ray tracing employing a specially developed three-dimensional mathematical model of the sensor and a respective computer program written in Visual Basic programming language. ${ }^{(15,16)}$ We assumed a transparent hemispherical detection element with refractive index $n_{\mathrm{e}}$, and two identical multimode optical fibers with the same refractive index of the core $n_{\text {core }}=n_{\mathrm{e}}$ and the same numerical aperture in air, $N A$. The fibers were perpendicular to the flat surface of the detection element. The light beam was modeled by a large number $(100,000)$ of geometrical rays. The Cartesian and angular coordinates of each ray at the end face of the lead-in optical fiber were assigned in a statistically random 
manner. For each ray, the point of incidence at the working surface and the reflected ray vector were determined. Ray tracing was repeated until each ray was either coupled to the lead-out optical fiber or lost. Then, the ray trajectories were plotted and the relative transmission was calculated as ${ }^{(10)}$

$$
T^{*}(n)=I_{\mathrm{r}}(n) / I_{\mathrm{rgas}},
$$

where $I_{\mathrm{r}}(n)$ is the light intensity at the sensor output (i.e., the light accepted by the leadout optical fiber, which is a function of the refractive index $n$ of the external medium), and $I_{\mathrm{r} \text { gas }}$ is the light intensity at the sensor output when the sensor is in the gas. Also, the signal was calculated as

$$
\mathfrak{R}=1 / T_{\text {LH2 }}^{*}=I_{\text {rgas }} / I_{\text {r LH2 }}
$$

and the intrinsic, non-fluid-dependent loss was calculated as

$$
A(\mathrm{~dB})=10 \log _{10}\left(I_{\mathrm{i}} / I_{\mathrm{r} n=1}\right),
$$

where $I_{\mathrm{i}}$ is the intensity of the input light (i.e., the light launched into the detection element from the lead-in optical fiber). The results of the numerical modeling of the sensor are given in $\S 3.1$.

\subsection{Experimental procedure}

The experimental procedure comprised the fabrication of the sensor and the investigation of its performance in the liquid-hydrogen storage tank.

The fiber-optic sensors were fabricated by fusing the ends of two multimode silica optical fibers and spherical segments of fused silica. The spherical working surface was achieved by heating the artifact to its softening temperature and the force of surface tension. On-line monitoring of the optical transmission and observation of the geometrical form of the artifact were performed during fabrication.

The sensor was monolithically integrated with two optical fibers with a length of about $50 \mathrm{~m}$ each. The two optical fibers connected the sensor to the optical transmitter, optical receiver, and the electronics associated with the measurement channel. The light source was an InGaAs light-emitting diode operating at $940 \mathrm{~nm}$. The photodetector was a Ge photodiode.

The liquid-hydrogen storage system consisted of a liquid-hydrogen tank, which was placed inside a tank containing liquid nitrogen. Both tanks were surrounded by a vacuum jacket, which protected the inner tank containing liquid hydrogen from the outside heat.

A total of seven fiber-optic sensors were installed at different vertical positions in the liquid-hydrogen tank. The lead-in and lead-out optical fibers passed all the way through the gas-tight couplings in the flanges of the two tanks to a remote electronic unit, which accommodated the optical transmitters, optical receivers, and other components of all measurement channels. The gas-tight couplings of the flanges could withstand an excess 
pressure of $4 \mathrm{MPa}$ at $20 \mathrm{~K}$. There were no optical connectors in the flanges. Therefore, there was no effect on the results of the measurements due to potentially unstable optical loss in the connectors.

In addition to the optical-fiber sensors, several loops of optical fibers with a length of about $0.5 \mathrm{~m}$ were installed in the liquid-hydrogen tank at different heights. The aim of this configuration was to evaluate the possible effects of the low temperature in the tank and the stress applied to the optical fibers in the gas-tight couplings on the attenuation of the optical fibers.

\section{Results}

\subsection{Numerical modeling of the sensor}

We analyzed the effect of various parameters on the relative transmission and intrinsic optical loss of the sensor. We considered nonpolarized, monochromatic light, an operational wavelength of $940 \mathrm{~nm}$, step-index multimode optical-fibers, a detection element made of fused silica with a refractive index of 1.45, an optical-fiber core made of the same material, and a refractive index of liquid hydrogen of $n_{\mathrm{LH} 2}=1.106$ at 940 $\mathrm{nm}$. Also, we considered a completely symmetrical sensor and optical fibers with equal parameters, because the fabrication of the sensor is much simpler in such a case.

The numerical simulation of light propagation in the sensor was performed in a range of external refractive indices from 1.0 to 1.2. We varied the diameter $\Phi$ in the range $0.05-0.6$ and the numerical aperture in the range 0.1-0.5. The optimum theoretical distance $\Lambda=0.707$ was used as a base, but we also varied this distance in the range $0.60-0.75$.

An example of light propagation in the transducer for a relatively small diameter of $\Phi=0.1$ with $N A=0.2$ is shown in Fig. 2 , and the respective relative transmission function is shown in Fig. 3, Curve 1. This case corresponds to a small, almost point source of light and a propagation pattern similar to that shown in Fig. 1. The efficiency of optical coupling of the two fibers is high (the intrinsic optical loss, $A$, is $3.7 \mathrm{~dB}$ ),

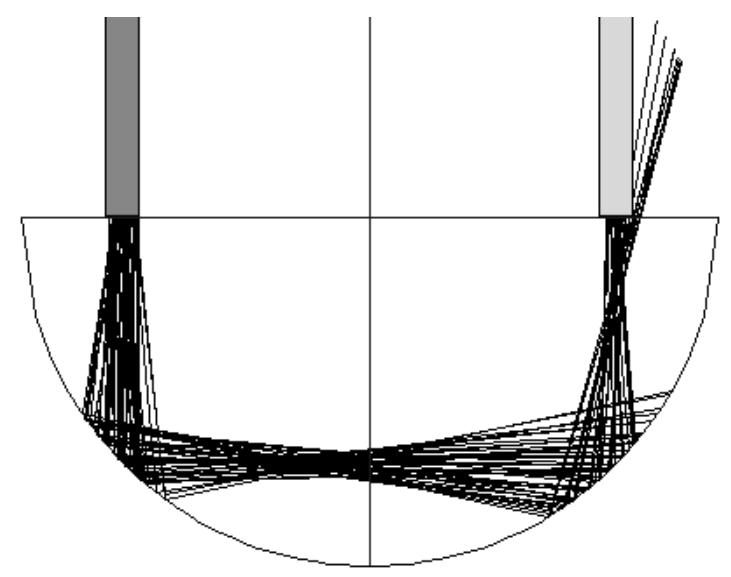

Fig. 2. Results of the numerical ray tracing for $\Lambda=0.707, \Phi=0.1$, and $N A=0.2$. 


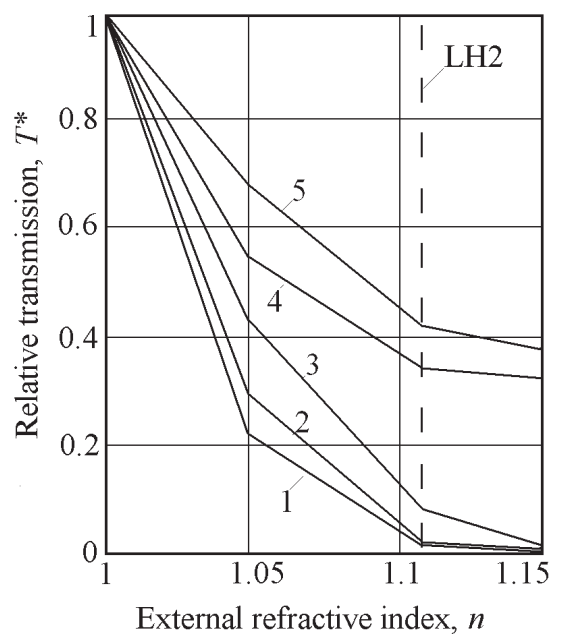

Fig. 3. Relative transmission $T^{*}$ vs external refractive index $n$ for $\Lambda=0.707$. Curve $1-\Phi=0.1$, $N A=0.2$; Curve $2-\Phi=0.3, N A=0.2$; Curve $3-\Phi=0.3, N A=0.4$; Curve $4-\Phi=0.5, N A=0.2$; Curve $5-\Phi=0.5, N A=0.4$.

the relative transmission in liquid hydrogen is 0.015 , and $\mathfrak{R}=18.2 \mathrm{~dB}$. However, the transverse dimension $W$ is large in comparison with the diameter of the optical fiber $D$. For instance, when $D=50 \mu \mathrm{m}, W=1 \mathrm{~mm}$.

The relative transmission functions calculated for some other combinations of parameters are also plotted in Fig. 3. These data show that the change in the relative transmission $T^{*}$ against the refractive index decreases with increasing diameter $\Phi$ and numerical aperture NA. This is due to the wide range of angles of incidence of light on the working surface. Many optical rays undergo total internal reflection at the working surface regardless of the refractive index of the external medium. For a large $N A$, these rays are coupled to the lead-out fiber and contribute to the parasitic, background light at the sensor output, which decreases the signal $\mathfrak{R}$.

The analysis showed a relatively small effect of $N A$ in the range $0.16-0.2$ on the signal $\mathfrak{R}$. In addition, we found that for a sufficiently small $N A$, a large diameter $\Phi$, and a suitable distance $\Lambda$, the optical transmission in the sensor occurs mainly due to the liquiddependent light, while other light is not coupled to the lead-out optical fiber, as shown in Fig. 4. Such a regime features a relatively large intrinsic optical loss. However, the lost light is not liquid-dependent. Therefore, this loss is useful because it is due to the nonliquid-dependent light, and therefore, it helps maintain a relatively large signal $\mathfrak{R}$ under excessive illumination of the working surface of the sensor by a wide optical beam.

The following are the results of the optimization of the sensor for the realistic parameter of $N A=0.18$ (we used this parameter because it corresponded to the optical fiber with the smallest NA that was available). The criterion for the optimality was the minimum of $T_{\mathrm{LH} 2}^{*}$. The relative transmission function for various values of $\Phi$ and the optimum distance $\Lambda$ for each parameter combination are shown in Fig. 5. The optimum 


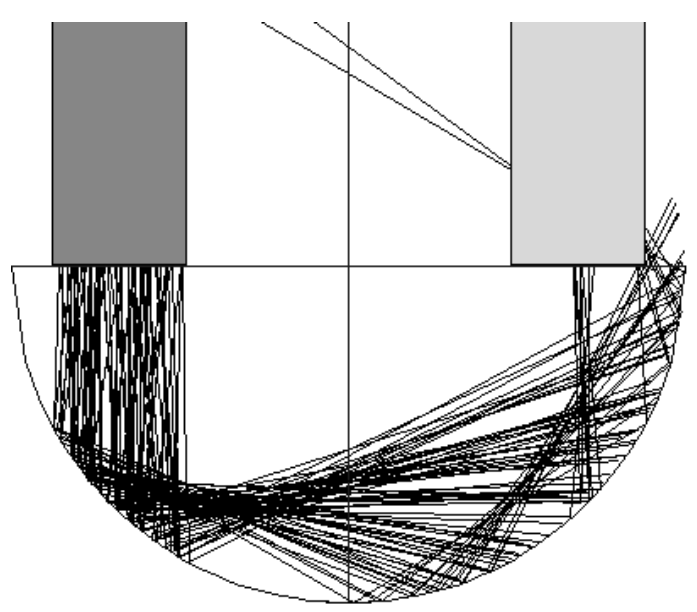

Fig. 4. Results of the numerical ray tracing for $\Lambda=0.68, \Phi=0.4$, and $N A=0.18$.

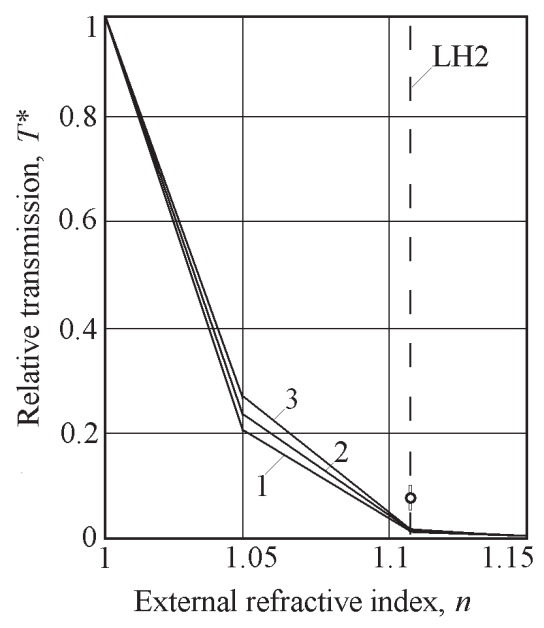

Fig. 5. Calculated relative transmission functions $T^{*}(n)$ for different diameters $\Phi$ (Curve $1-\Phi$ $=0.1$, Curve $2-\Phi=0.3$, Curve $3-\Phi=0.5), N A=0.18$, and the optimum parameter $\Lambda$; $\left({ }^{\circ}\right)-$ the observed relative transmission.

distance $\Lambda$ was obtained by a numerical experiment. $\Lambda$ varied quasi-linearly in the range 0.69 -0.60 as the diameter $\Phi$ ranged from 0.05 to 0.6 . The graphs of the relative transmission $T_{\text {LH2 }}^{*}$ and the intrinsic optical loss $A$ against $\Phi$ for the optimum distance $\Lambda$ are shown in Figs. 6 and 7, respectively.

The data in Fig. 5 show that for a reasonably small and realistic value of NA and a suitable parameter $\Lambda$, the diameter $\Phi$ has a moderate effect on the relative transmission function $T^{*}(n)$. The related change in signal intensity is only from about $19 \mathrm{~dB}$ to $15 \mathrm{~dB}$ 


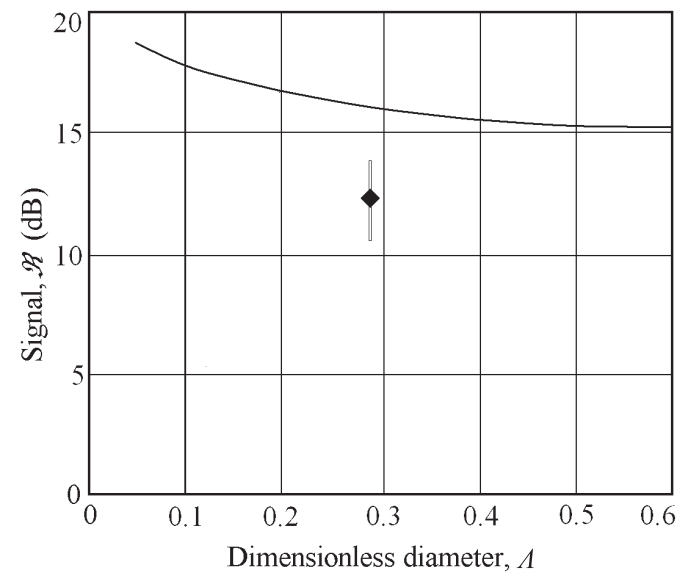

Fig. 6. Theoretical limit of the signal $\mathfrak{R}$ against the dimensionless diameter $\Phi$ for the optimum parameter $\Lambda ;(\bullet)$ - the observed signal.

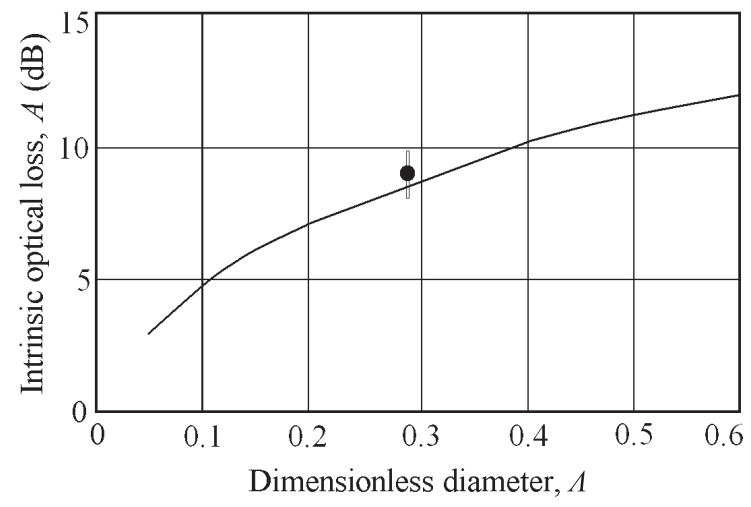

Fig. 7. Calculated intrinsic optical loss $A$ vs dimensionless diameter $\Phi$ for the optimum parameter $\Lambda$; $(\bullet)$ the observed intrinsic optical loss.

(Fig. 6). The intrinsic optical loss increases monotonically from about $3 \mathrm{~dB}$ to $12 \mathrm{~dB}$ in the same range of $\Phi$.

The relatively small effect of the diameter $D$ on the signal $\mathfrak{R}$ allows us to design the present sensor with a relatively large value of $\Phi(0.2<\Phi \leq 0.6)$ while satisfying the specified conditions: a small $N A$ and the optimum $\Lambda$, which reduces the transverse dimension of the sensor $W$.

\subsection{Experimental results}

A group of seven fiber-optic sensors was fabricated from spherical segments made of fused synthetic silica and optical fibers with a $\mathrm{SiO}_{2}$ core, $\mathrm{B}_{2} \mathrm{O}_{3}-\mathrm{SiO}_{2}$ cladding of internal 
and external diameters $D=50 \mu \mathrm{m}$ and $d=125 \mu \mathrm{m}$, respectively, and $N A=0.18$. The transverse dimension of the sensor was $W=0.35 \mathrm{~mm}(\Phi=0.29)$ and the parameter was $\Lambda=0.68$. The sensors were installed in the liquid-hydrogen storage tank, which was half-filled and then partially drained. The data runs consisted of first positioning the transducers in the bulk liquid and then cycling them though the liquid interface. Sensor outputs were recorded on a digital oscilloscope and stored in a computer. The analysis was performed to evaluate the response of the sensors to the change in the external medium from liquid hydrogen to gaseous hydrogen and vice versa. Five liquid-hydrogen filling and draining tests were conducted in which the optical-fiber transducers were used to indicate the liquid interface in the tank.

When passing through the liquid interface, the average signal of the sensors was $11.75 \mathrm{~dB}\left(T_{\mathrm{LH} 2}^{*}=0.067\right)$. The mean intrinsic optical loss $A$ was $8.5 \mathrm{~dB}$. The optical fibers with no sensors showed a change in optical transmission of less than $0.2 \mathrm{~dB}$ when the temperature changed from room temperature to approximately $20 \mathrm{~K}$ in the liquidhydrogen tank of the storage system.

\section{Discussion}

We showed theoretically and experimentally that the optical-fiber refractometric sensor with a hemispherical detection element made of fused silica can be optimized for the detection of the liquid interface in liquid-hydrogen tanks. The sensor, which is monolithically integrated with the lead-in and lead-out optical fibers, is well suited for operation at very low cryogenic temperatures. Also, it was found that the transverse dimensions of the sensor could be minimized without a significant decrease in sensitivity if optical fibers with a sufficiently small numerical aperture were used and when the position of the optical fibers in the sensor resulted in a significant loss of non-liquiddependent light. Small transverse dimensions of the sensor offer many advantages in a realistic operational environment, such as the reduced retention of liquid on its surface in a form of a film or a drop.

The observed signal intensity is smaller than its theoretically predicted value, whereas the intrinsic optical loss almost coincides with the theoretical prediction. We attribute the difference between the observed and predicted signal intensities to the factors that were not considered in the theoretical analysis, such as possible deviations of the actual form of the sensor from the ideal geometrical form, nonhomogeneity of the sensor material, and the scattering and diffraction of light. Nevertheless, the experimentally observed signal was sufficiently large for the reliable discrimination between the liquid hydrogen and the gaseous hydrogen above it in the storage tank.

The relatively large intrinsic optical loss of this sensor (3-12 dB) does not present a significant problem provided the power budget of the measurement system is larger by several orders of magnitude.

This sensor can be used as a point device, in pairs, or in multipoint arrays, such as those used in discrete liquid-level sensors. Prospective applications include liquidhydrogen level-sensing in hydrogen-powered cars, aircraft, space vehicles, and liquidhydrogen production and storage facilities. 
In conclusion, the successful operation of a fiber-optic refractometric sensor as an indicator of the liquid interface in a liquid-hydrogen tank was demonstrated for the first time, to the best of our knowledge. The advantages of this sensor in comparison with electrical sensors are the smaller heat ingress in the liquid-hydrogen tank, its intrinsic safety, and its potentially low cost.

\section{Acknowledgments}

The authors acknowledge the support of the Faculty of Engineering and General Directorate of Academic Personnel (DGAPA) of the National Autonomous University of Mexico in the form of Research Projects IN100502, IN106906, and PE102305, as well as the support of the National Science and Technology Council of Mexico (CONACYT) in the form of Research Grant 026106 (51058).

\section{References}

1 G. P. Sutton: Rocket Propulsion Elements: An Introduction to the Engineering of Rockets, 7th ed. (Wiley, New York, 1992).

2 R. Wurster, H. Knorr and W. Prümm: Proc. 9th Canadian Hydrogen Conf. (Vancouver, Canada, 1999) p. 70.

3 G. Krainz, G. Bartlok, P. Bodner, P. Casapicola, Ch. Doeller, F. Hofmeister, E. Neubacher and S. Kasthurirengan: AIP Conf. Proc. 710 (2004) 35.

4 R. Karunanithi and S. Kasthurirengan: Adv. Cryog. Eng. 48 (2000) 1803.

5 M. Haberbush: Adv. Cryog. Eng. 47 (2002) 1292.

6 Ch. Haberstroh and G. A. Zick: AIP Conf. Proc. 823 (2006) 679.

7 C. Yang, S. Chen and A. Kazemi: Proc. SPIE 4204 (2001) 206.

8 V. Svirid, S. Khotiaintsev and P. Swart: Opt. Eng. 41 (2002) 779.

9 V. Svirid, S. Khotiaintsev and P. Swart: Opt. Eng. 42 (2003) 1383.

10 K. Romo-Medrano, S. Khotiaintsev and V. Garcia-Garduño: Meas. Sci. Technol. 15 (2004) 1568.

11 K. Romo Medrano and S. Khotiaintsev: Meas. Sci. Technol. 17 (2006) 998.

12 CRC Handbook of Chemistry and Physics, 63rd ed. (CRC Press, Boca Raton, 1982) Chap. E.

13 M. J. Adams: An Introduction to Optical Waveguides (John Wiley and Sons, Chichester, 1981) Chap. 2.

14 M. Medhat, S. Y. El-Zaiat, A. Radi and M. F. Omar: J. Opt. A: Pure Appl. Opt. 4 (2002) 174.

15 V. Svirid: Optical-Fiber Refractometric Sensors, PhD Thesis (National Autonomous University of Mexico, Mexico, 2001) Chap. 4 (in Spanish).

16 K. Khotiaintsev-Umanets: Development of Software for Numerical Simulation of OpticalFiber Refractometric Sensors, B. Sc. Thesis (Puebla Autonomous University, Puebla, 2005) Chap. 3 (in Spanish). 